\title{
Periodically Nonuniform Sampling of Bandpass Signals
}

\author{
Yuan-Pei Lin, Member, IEEE, and P. P. Vaidyanathan, Fellow, IEEE
}

\begin{abstract}
It is known that a continuous time signal $x(t)$ with Fourier transform $X(\nu)$ band-limited to $|\nu|<\Theta / 2$ can be reconstructed from its samples $x\left(T_{0} n\right)$ with $T_{0}=2 \pi / \Theta$. In the case that $X(\nu)$ consists of two bands and is band-limited to $\nu_{0}<|\nu|<\nu_{0}+\Theta / 2$, successful reconstruction of $x(t)$ from $x\left(T_{0} n\right)$ requires an additional condition on the band positions. When the two bands are not located properly, Kohlenberg showed that we can use two sets of uniform samples, $x\left(2 T_{0} n\right)$ and $x\left(2 T_{0} n+d_{1}\right)$, with average sampling period $T_{0}$, to recover $x(t)$. Because two sets of uniform samples are employed, this sampling scheme is called Periodically Nonuniform Sampling of second order $[\operatorname{PNS}(2)]$. In this paper, we show that $\operatorname{PNS}(2)$ can be generalized and applied to a wider class. Also, Periodically Nonuniform Sampling of $L$ th-order $[\operatorname{PNS}(L)]$ will be developed and used to recover a broader class of band-limited signals. Further generalizations will be made to the two-dimensional case and discrete time case.
\end{abstract}

\section{INTRODUCTION}

I $\mathrm{T}$ is well known that a continuous time band-limited signal $x(t)$ can be reconstructed from its samples. If $x(t)$ has bandwidth $\Theta$ as shown in Fig. 1, $x(t)$ can be recovered from its samples $x(n T)$ as long as the sampling period $T \leq T_{0}$, where $T_{0}=2 \pi / \Theta$. A lowpass interpolation filter $F(\nu)$ as shown in Fig. 2 can be used to recover $x(t)$ (Fig. 3). Suppose now $x(t)$ has total bandwidth $\Theta$, but $X(\nu)$ consists of two bands as in Fig. 4. Successful reconstruction from $x\left(n T_{0}\right)$ depends on the relative positions of these two bands [1]. A necessary and sufficient condition is that the frequency $\nu_{0}$ (indicated in Fig. 4) must be an integer multiple of $\Theta / 2$. More generally, it can be shown that a much wider class of signals with total bandwidth $\Theta$ can be recovered from samples at $n T_{0}$. To be more specific, define the support of $X(\nu)$ [denoted by $\mathcal{S}(X)$ ] to be the set of frequencies for which $X(\nu) \neq 0$. Then $x(t)$ can be obtained from $x\left(n T_{0}\right)$ if and only if no two frequencies in $\mathcal{S}(X)$ overlap under modulo $\Theta$ operation [2]-[5]. Such signals are called aliasfree $\left(T_{0}\right)$ and their supports are referred to as aliasfree $\left(T_{0}\right)$ zones.

When the two bands of $X(\nu)$ (Fig. 4) were not properly located, Kohlenberg [6] proposed a periodically nonuniform sampling approach to recover $x(t)$. Two sets of samples, $x(n T)$ and $x\left(n T+d_{1}\right)$, where $T=2 T_{0}$, as shown in

Manuscript received August 15, 1996; revised July 3, 1997. This work was supported in part by ONR under Grant N00014-93-0231, and by Tektronix, Inc. This paper was recommended by Associate Editor N. K. Bose.

Y. P. Lin was with the Department of Electrical Engineering, California Institute of Technology, Pasadena, CA 91125 USA. She is now with National Chiao-Tung University, Hsinchu, Taiwan, R.O.C.

P. P. Vaidyanathan is with the Department of Electrical Engineering, California Institute of Technology, Pasadena, CA 91125 USA.

Publisher Item Identifier S 1057-7130(98)00774-5.

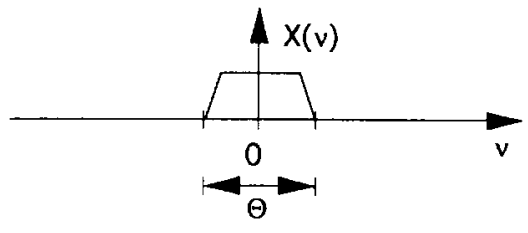

Fig. 1. Band-limited signal with bandwidth $\Theta$.

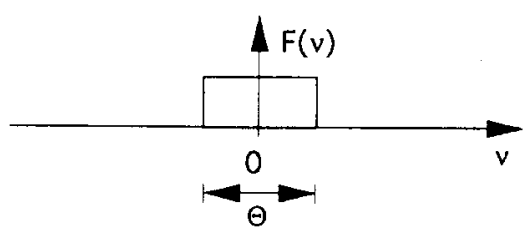

Fig. 2. A lowpass interpolation filter.

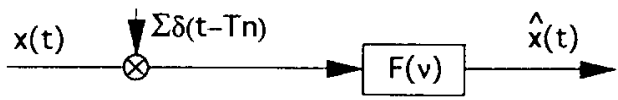

Fig. 3. Reconstruction of $x(t)$ from uniform samples by using a lowpass filter $F(\nu)$.

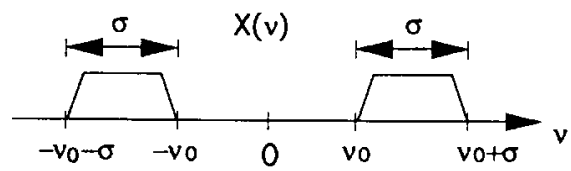

Fig. 4. Band-limited signal with two bands and total bandwidth $\Theta=2 \sigma$.

Fig. 5, are used; the average sampling rate is still $\Theta$. This sampling and reconstruction scheme can be described by the diagram shown in Fig. 6. It is shown in [6] that $x(t)$ can be successfully reconstructed by properly choosing $d_{1}$ and the synthesis filters $f_{0}(t)$ and $f_{1}(t)$. This is called periodically nonuniform sampling of second order [PNS(2)] [7], for there are two sets of uniform samples involved. Recently, general $L$ th-order periodically nonuniform sampling $[\mathrm{PNS}(L)]$ and reconstruction (Fig. 7) for such two-bands signals has been considered in [8]. Using $\operatorname{PNS}(L)$ sampling allows more freedom in choosing the locations of the samples. Reconstruction of two-band signals from the samples of $L$ filtered outputs are studied in [9] and [10]; conditions on the $L$ filters for reconstruction are presented. These conditions are extended in [11] for the more general class, namely those whose frequency support consist of several intervals. The reconstruction of signals from nonuniformly sampled versions has also been addressed in [12] and [13]. 


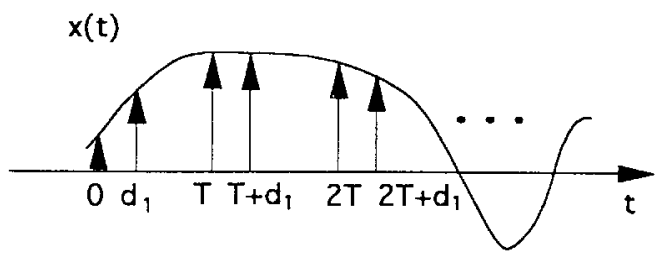

Fig. 5. Illustration of second-order periodical nonuniform sampling.

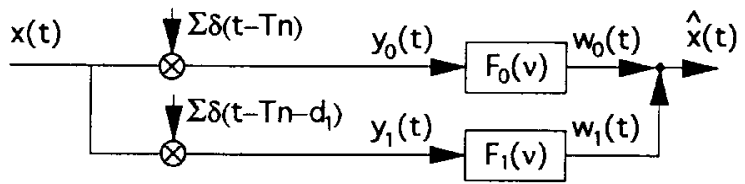

Fig. 6. Reconstruction of $x(t)$ using periodical nonuniform sampling of second order, where $T=2 \pi / \sigma$.

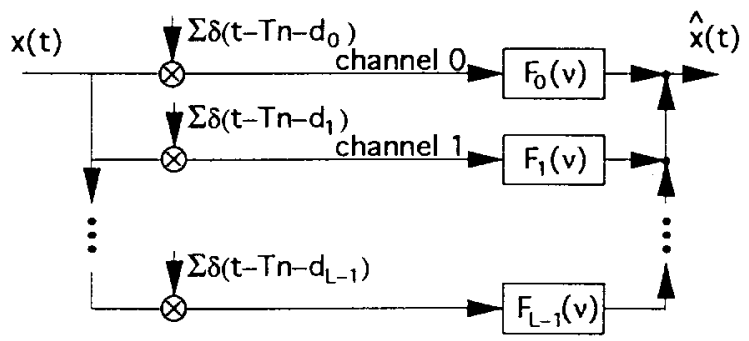

Fig. 7. Reconstruction of $x(t)$ using periodically nonuniform sampling of $L$ th order.

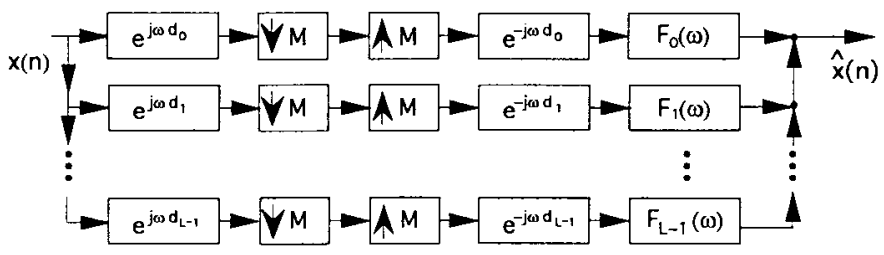

Fig. 8. Periodically nonuniform sampling and reconstruction in discrete time case.

In the discrete time case, sampling is replaced by decimation. Periodically nonuniform sampling of $L$ th order retains $L$ sets of samples, $x\left(M n+d_{0}\right), x\left(M n+d_{1}\right), \cdots, x(M n+$ $\left.d_{L-1}\right)$, for some integer $M$. The decimated signal $x(M n+$ $d$ ) is called the $d$ th polyphase component of $x(n)$ [14]; the operation of $\operatorname{PNS}(L)$ sampling retains the $d_{0}$ th, $d_{1}$ th, $\cdots, d_{L-1}$ th polyphase components. In [15] and [16], PNS $(L)$ sampling and reconstruction (Fig. 8) has been considered for a very restricted subclass of $L$-band signals. The subclasses addressed therein are those signals whose frequency supports are the union of $L$ bands, each band with bandwidth $2 \pi / M$ and band edges at integer multiples of $2 \pi / M$, e.g., the one shown in Fig. 9 (the definition of $L$-band signal here is different from that in [13]). It is shown in [16] that such an $L$-band signal $x(n)$ can be reconstructed from its first $L$ polyphase components, i.e., $x(M n), x(M n+1), \cdots, x(M n+L-1)$.

In this paper, we first generalize the results in [6] and [16] to a significantly wider class of signals in terms of signal frequency supports. The supports considered in [6] are the union of two intervals, each of length $\sigma$. Generalization will be made

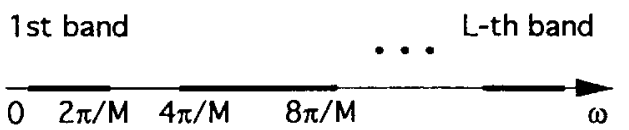

Fig. 9. An $L$-band signal with restricted band edges.

to the class of $U(T, 2)$ signals [17], which is the collection of signals whose supports are the union of two nonoverlapping aliasfree $(T)$ sets. ${ }^{1}$ We will show that this class of signals can be reconstructed from PNS(2) samples. An extension of this result to the more general $U(T, L)$ class using $L$ th-order periodically nonuniform sampling scheme will be developed. We will see that the 2-D counterpart of this can be shown in a similar manner. Furthermore, the discrete time version of these will be addressed. In this regard, we find that 1-D discrete time $U(M, L)$ signals can always be reconstructed from their first $L$ polyphase components. However, in 2-D discrete time case, only a subclass of $U(\boldsymbol{M}, L)$ signals allows reconstruction from $L$ polyphase components.

\section{A. Paper Outline}

In Section II-A, we provide a review of Kohlenberg's results [6]. The generalizations presented in later sections depend to some extent on this review. In Section II-B, we will show that the reconstruction of two band signals in [6] is stable, although the reconstruction filters are not stable in the Bounded Input Bounded Output sense. The definition of stable reconstruction will also be given in Section II-B. The results in [6] are generalized for the more general continuous time $U(T, L)$ signals. The generalization to discrete time $U(M, L)$ signals is straightforward, and the main results will be mentioned briefly in Section III. We present, in Section IV, the 2-D version (continuous time) of the theorem given in Section III. Generalizations in this case follow routinely. However, the 2-D discrete time case exhibits some unusual behavior and will therefore be addressed in greater detail in Section V. A conclusion is given in Section VI. Some preliminary versions of the results derived here have been presented at recent conferences [17], [18].

\section{B. Notations}

1) Boldfaced lower case letters are used to represent vectors, and boldfaced upper case letters are reserved for matrices. The notations $\boldsymbol{A}^{T}$ and $|\boldsymbol{A}|$ represent the transpose of $\boldsymbol{A}$ and the absolute value of the determinant of A.

2) Fourier transforms. The Fourier transform of a 1-D continuous time signal $x(t)$ is denoted by $X(\nu)$ [1]. For a 2-D signal $x(\boldsymbol{t})$, where $\boldsymbol{t}$ is a $2 \times 1$ vector, the Fourier transform is $X(\boldsymbol{\nu})$, where $\boldsymbol{\nu}$ is a $2 \times 1$ frequency vector. For discrete time signals, the Fourier transforms of a 1-D sequence $x(n)$ and a 2-D signal $x(\boldsymbol{n})$ are denoted, respectively, by $X(\omega)$ and $X(\boldsymbol{\omega})$.

3) The support of $X(\nu)$ [denoted by $\mathcal{S}(X)$ ] is defined as the set of frequencies for which $X(\nu) \neq 0$.

\footnotetext{
${ }^{1}$ Throughout this paper, we will assume that aliasfree $(T)$ sets contain only finitely many intervals.
} 
4) Aliasfree $(T)$ signals and aliasfree $(T)$ zones. A signal is called aliasfree $(T)$ if the sampling of $x(t)$ with period $T$ does not create aliasing. In this case, the support of $X(\nu)$ is referred to as an aliasfree $(T)$ zone. Equivalently, an aliasfree $(T)$ zone can be defined as a set such that no two frequencies in the set overlap under modulo $2 \pi / T$ operation.

5) The notation $U(T, L)$ represents the collection of signals whose frequency supports are the union of $L$ nonoverlapping aliasfree $(T)$ sets.

6) The characteristic function $\chi_{S}(\nu)$ of a set $S$ is defined as

$$
\chi_{S}(\nu)= \begin{cases}1, & \nu \in S \\ 0, & \text { otherwise }\end{cases}
$$

\section{RECONSTRUCTION OF TWO-BAND SIGNALS FROM PNS(2) SAMPLES}

\section{A. Reconstruction from Periodically Nonuniform Samples [6]}

Consider the two-band signal in Fig. 4 and the sampling scheme in Fig. 6. In each channel, the sampling period is $T=2 \pi / \sigma$ and the average sampling period is $T / 2$. We first derive a general expression for the recombined signal in terms of the input and synthesis filters. The Fourier transforms of $y_{0}(\nu)$ and $y_{1}(\nu)$ (as indicated in Fig. 6) in terms of the inputs are, respectively,

$$
Y_{0}(\nu)=\frac{1}{T} \sum_{k \in \mathcal{Z}} X(\nu-k \sigma)
$$

and

$$
Y_{1}(\nu)=\frac{1}{T} \sum_{k \in \mathcal{Z}} X(\nu-k \sigma) e^{-j k d_{1} \sigma}
$$

where $T=2 \pi / \sigma$.

The recombined signal is $\hat{X}(\nu)=Y_{0}(\nu) F_{0}(\nu)+Y_{1}(\nu) F_{1}(\nu)$, and we have

$$
\hat{X}(\nu)=\frac{1}{T} \sum_{k \in \mathcal{Z}} X(\nu-k \sigma)\left[F_{0}(\nu)+e^{-j k d_{1} \sigma} F_{1}(\nu)\right] .
$$

As the total bandwidth of $X(\nu)$ is $2 \sigma$ and the sampling period is $T$ in each channel, aliasing occurs in $Y_{0}(\nu)$ and $Y_{1}(\nu)$. However, with a priori knowledge of the band position of $X(\nu)$, a proper choice of $d_{1}$ and synthesis filters will allow us to cancel aliasing. The value of $d_{1}$ depends on the band positions of $X(\nu)$.

To see this, let $X_{0}(\nu)$ be the part of $X(\nu)$ restricted to positive frequencies, i.e.,

$$
X_{0}(\nu)= \begin{cases}X(\nu), & \nu \geq 0 \\ 0, & \text { otherwise }\end{cases}
$$

Let $X_{1}(\nu)$ be the part of $X(\nu)$ restricted to negative frequencies. Then both $X_{0}(\nu)$ and $X_{1}(\nu)$ are aliasfree $(T)$ signals. By (1), the signals $Y_{0}(\nu)$ and $Y_{1}(\nu)$ consist of repeated copies of $X(\nu)$, i.e., repeated copies of $X_{0}(\nu)$ and $X_{1}(\nu)$. Because the bandwidth of each $X_{i}(\nu)$ is $\sigma=2 \pi / T$ and the sampling period is $T$ in each channel, the repeated copies of each $X_{i}(\nu)$ will fill the whole frequency line.

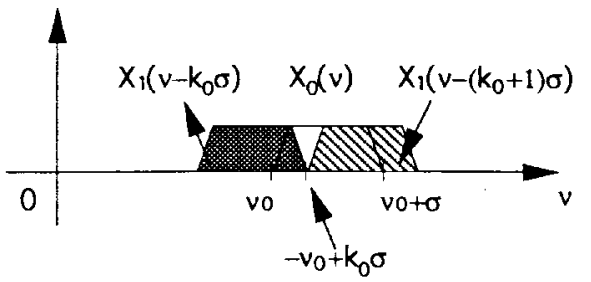

Fig. 10. Shifted copies of $X_{1}(\nu)$ and relative location to $X_{0}(\nu)$.

Among the shifted copies of $X_{1}(\nu)$, we see from Fig. 10 that only $X_{1}\left(\nu-k_{0} \sigma\right)$ and $X_{1}\left[\nu-\left(k_{0}+1\right) \sigma\right]$ contribute to $Y_{\ell}(\nu)$ in the interval $\left(\nu_{0}, \nu_{0}+\sigma\right)$, where $k_{0}=\left\lceil 2 \nu_{0} / \sigma\right\rceil(\lceil a\rceil$ is the smallest integer greater or equal to $a$ ). In particular,

$$
T Y_{0}(\nu)=\left\{\begin{aligned}
& X_{0}(\nu)+X_{1}\left(\nu-k_{0} \sigma\right), \\
& \nu \in\left(\nu_{0},-\nu_{0}+k_{0} \sigma\right) \\
& X_{0}(\nu)+X_{1}\left[\nu-\left(k_{0}+1\right) \sigma\right] \\
& \nu \in\left(-\nu_{0}+k_{0} \sigma, \nu_{0}+\sigma\right) .
\end{aligned}\right.
$$

Because $X_{0}(\nu)$ and $X_{1}(\nu)$ are nonoverlapping, $Y_{0}(\nu)$ can be rewritten as

$$
T Y_{0}(\nu)=\left\{\begin{array}{c}
X(\nu)+X\left(\nu-k_{0} \sigma\right) \\
\nu \in\left(\nu_{0},-\nu_{0}+k_{0} \sigma\right) \\
X(\nu)+X\left[\nu-\left(k_{0}+1\right) \sigma\right] \\
\nu \in\left(-\nu_{0}+k_{0} \sigma, \nu_{0}+\sigma\right)
\end{array}\right.
$$

A similar expression can be derived for $Y_{0}(\nu)$ for the interval $\left(-\nu_{0}-\sigma,-\nu_{0}\right)$. Let $\beta(\nu)$ be an integer function defined as

$$
\beta(\nu)= \begin{cases}k_{0}, & \nu \in\left(\nu_{0},-\nu_{0}+k_{0} \sigma\right) \\ k_{0}+1, & \nu \in\left(-\nu_{0}+k_{0} \sigma, \nu_{0}+\sigma\right)\end{cases}
$$

and

$$
\beta(\nu)=-\beta(-\nu) \quad[\text { Fig. 11(a) }] .
$$

Then we can write (2) as

$$
T Y_{0}(\nu)=X(\nu)+X[\nu-\beta(\nu) \sigma], \quad \text { for } \nu \in \mathcal{S}(X)
$$

where $\mathcal{S}(X)$, the support of $X(\nu)$, is as defined in Section I-B. Similarly,

$T Y_{1}(\nu)=X(\nu)+X[\nu-\beta(\nu) \sigma] e^{-j \beta(\nu) d_{1} \sigma}, \quad$ for $\nu \in \mathcal{S}(X)$.

Using the above two expressions for $Y_{0}(\nu)$ and $Y_{1}(\nu)$, we have

$$
\begin{gathered}
T \hat{X}(\nu)=X(\nu)\left[F_{0}(\nu)+F_{1}(\nu)\right]+X[\nu-\beta(\nu) \sigma] \\
\cdot\left[F_{0}(\nu)+e^{-j \beta(\nu) d_{1} \sigma} F_{1}(\nu)\right], \\
\text { for } \nu \in \mathcal{S}(X) .
\end{gathered}
$$

From (2), we see that if $F_{0}(\nu)=F_{1}(\nu)=0$ for $\nu \notin \mathcal{S}(X)$, then $\hat{X}(\nu)=0$, for $\nu \notin \mathcal{S}(X)$. It follows that $\hat{x}(t)=x(t)$ if the following conditions are satisfied.

$$
F_{0}(\nu)=F_{1}(\nu)=0, \quad \nu \notin \mathcal{S}(X),
$$

and

$$
\left\{\begin{array}{l}
F_{0}(\nu)+F_{1}(\nu)=T, \\
F_{0}(\nu)+e^{-j \beta(\nu) d_{1} \sigma} F_{1}(\nu)=0
\end{array} \quad \nu \in \mathcal{S}(X) .\right.
$$

We can find $F_{0}(\nu)$ and $F_{1}(\nu)$ that satisfy (5) if there exists $d_{1}$ such that $e^{-j \beta(\nu) d_{1} \sigma} \neq 1$ for every $\nu \in \mathcal{S}(X)$. This requires that $d_{1} \beta(\nu) / T$ is not an integer for any $\nu \in \mathcal{S}(X)$. Since $\beta(\nu)$ takes on only four values, $\pm k_{0}$ and $\pm\left(k_{0}+1\right)$, we can 


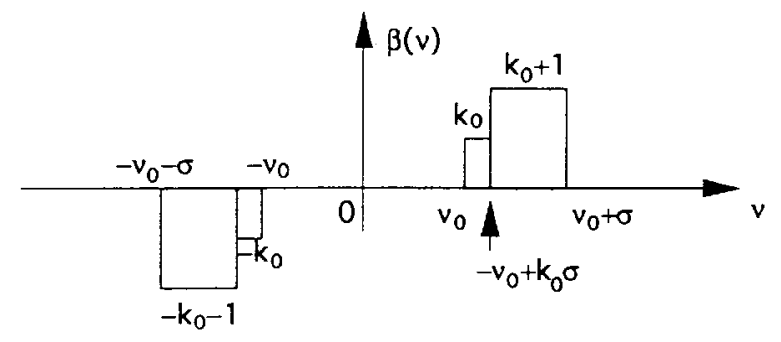

(a)

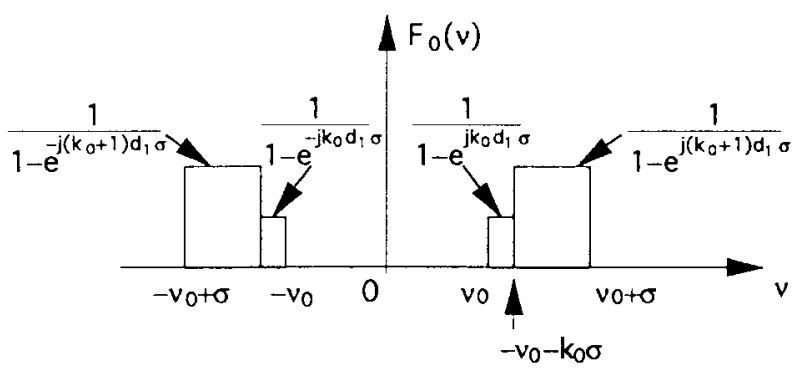

(b)

Fig. 11. Sketches of (a) $\beta(\nu)$ and (b) $F_{0}(\nu)$.

always find $d_{1}$ such that $e^{-j \beta(\nu) d_{1} \sigma} \neq 1$. In particular, we can find rational $d_{1}$ that satisfies this condition. For example, choose $d_{1}=1 / p$, where $p$ is an integer coprime with $k_{0}$ and $k_{0}+1$, then $e^{-j \beta(\nu) d_{1} \sigma} \neq 1$ for all $\nu$. When $e^{-j \beta(\nu) d_{1} \sigma} \neq 1$, we can solve (5) and obtain the synthesis filters. Therefore, we can always recover a two-band signal $x(t)$ from the sequences $x(T n)$ and $x\left(T n+d_{1}\right)$. Summarizing, we have the following theorem [6].

Theorem 1: Let $x(t)$ be a two-band signal, each band of length $\sigma$, as shown in Fig. 4. Then $x(t)$ can be reconstructed from $x(n T)$ and $x\left(n T+d_{1}\right), T=2 \pi / \sigma$, through the following formula.

$$
x(t)=\sum_{n \in \mathcal{Z}} x(n T) f_{0}(t-n T)+x\left(n T+d_{1}\right) f_{1}(t-n T)
$$

where $d_{1}$ is such that $d_{1} \beta(\nu) / T$ is not an integer for any $\nu \in \mathcal{S}(X)$. The synthesis filters $F_{\ell}(\nu)$ are given by

$F_{0}(\nu)=\frac{T}{1-e^{j \beta(\nu) d_{1} \sigma}} \chi_{s(X)}(\nu) \quad$ and $F_{1}(\nu)=F_{0}(-\nu)$

where $\beta(\nu)$ is as defined in (3) and the characteristic function $\chi_{S}(\nu)$ of a set $S$ is as defined in Section I-B.

Remark on the Synthesis Filters: Observe that the function $\beta(\nu)$ defined in (3) is piecewise constant [Fig. 11(a)]. The synthesis filters given in (7) are functions of $\beta(\nu)$ and are hence piecewise constant [Fig. 11(b)]. The synthesis filters $F_{\ell}(\nu)$ are constant with four different heights in four intervals. This leads to the property that the synthesis filters can be viewed as a linear combination of four ideal filters, each with bandwidth $<\sigma$. This observation will be useful in showing the stability of the reconstruction in the next subsection.

\section{B. Stability of Reconstruction}

Consider a signal $x(t)$ that can be reconstructed from a sequence $x_{n}$ with $\left\|x_{n}\right\|<\infty$ through the following equation.

$$
x(t)=\sum_{n \in \mathcal{Z}} x_{n} f(t-n T)
$$

pointwise for each $t$. Suppose we add an error sequence $e_{n}$ to $x_{n}$ and the corresponding reconstructed signal is $x(t)+e(t)$. Then the reconstruction is stable if a small $\sum_{n \in \mathcal{Z}}\left|e_{n}\right|^{2}$ incurs only a correspondingly small (in some sense) error $e(t)$. More precisely, the reconstruction is pointwise stable if there exists $\alpha_{t}$ independent of $x_{n}$ such that

$$
|e(t)| \leq \alpha_{t}\left\|e_{n}\right\|
$$

where

$$
\left\|e_{n}\right\|=\left(\sum_{n \in \mathcal{Z}}\left|e_{n}\right|^{2}\right)^{1 / 2}\left(2 \text {-norm of } e_{n}\right)
$$

Observe that by Cauchy inequality, (8) yields

$$
|x(t)| \leq\left\|x_{n}|\|\mid f(t-n T)\| .\right.
$$

For the case where $F(\nu)$ is an ideal brick-wall filter with bandwidth $2 \pi / T$ (Shannon reconstruction)

$$
\|f(t-n T)\|=1 \text {. }
$$

In this case,

$$
|x(t)| \leq\left\|x_{n}\right\|
$$

holds for arbitrary sequence $x_{n}$ with $\left\|x_{n}\right\|<\infty$. From (8), we have $e(t)=\sum_{n \in \mathcal{Z}} e_{n} f(t-n T)$ by linearity; hence $|e(t)| \leq\left\|e_{n}\right\|$. So the reconstruction is pointwise stable.

Returning now to the reconstruction scheme in Fig. 6, the signals $w_{0}(t)$ and $w_{1}(t)$ are

$$
w_{0}(t)=\sum_{n \in \mathcal{Z}} x(n T) f_{0}(t-n T)
$$

and

$$
w_{1}(t)=\sum_{n \in \mathcal{Z}} x\left(n T+d_{1}\right) f_{1}(t-n T)
$$

Using the fact that each synthesis filter is a sum of ideal filters, it follows that

$$
\left|w_{0}(t)\right| \leq A_{0}\|x(n T)\| \quad \text { and } \quad\left|w_{1}(t)\right| \leq A_{1}\left\|x\left(n T+d_{1}\right)\right\| .
$$

As $x(t)=w_{0}(t)+w_{1}(t)$, we have

$|x(t)| \leq\left|w_{0}(t)\right|+\left|w_{1}(t)\right| \leq A_{0}|| x(n T)\left\|+A_{1}|| x\left(n T+d_{1}\right)\right\|$

and the reconstruction is pointwise stable. 


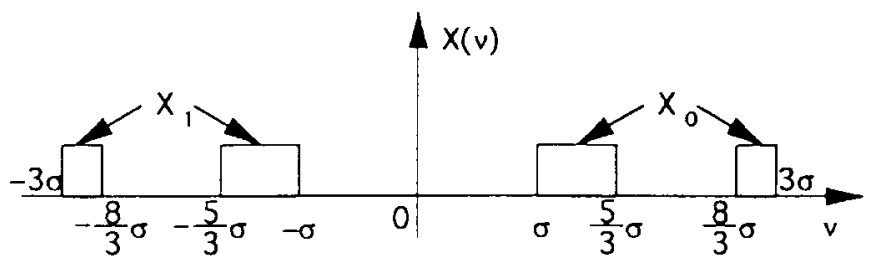

(a)

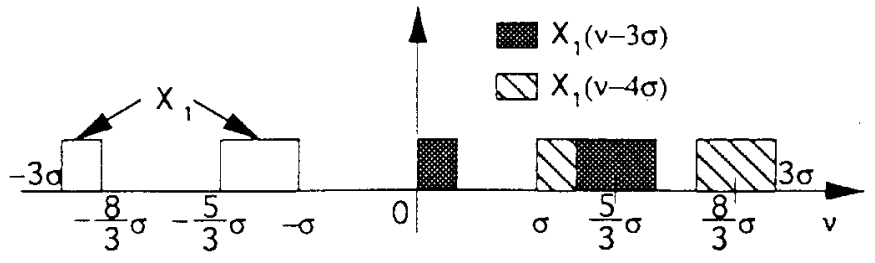

(b)

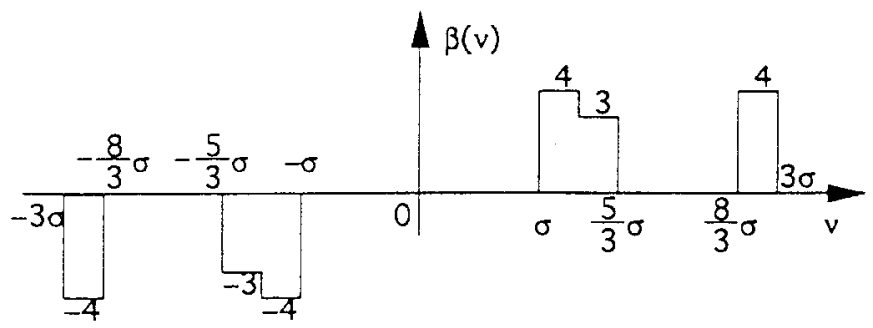

(c)

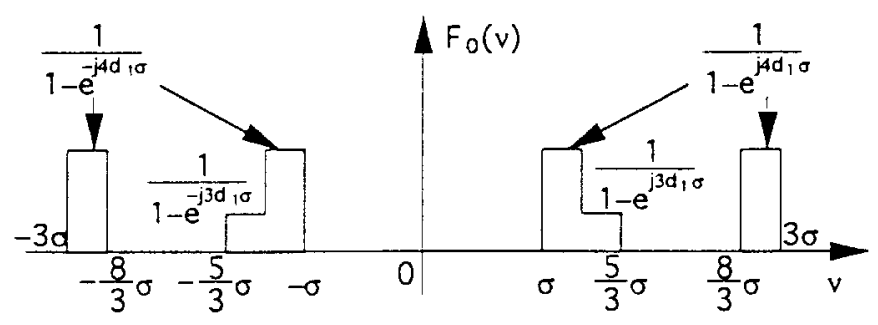

(d)

Fig. 12. Example 1. (a) Support of $X(\nu)$, (b) shifts of $X_{1}(\nu)$, (c) sketch of $\beta(\nu)$, and (d) sketch of $F_{0}(\nu)$.

\section{Periodically Nonuniform SAmpling OF $L$ TH ORDER}

The signals considered in [6] and [8] have two bands as shown Fig. 4; the frequency support is the union of two disjoint intervals. The two intervals (call them $S_{0}$ and $S_{1}$ ) have length $\sigma$ and are therefore aliasfree $(T)$ zones, where $T=2 \pi / \sigma$. In this section, we will generalize the results in [6] and [8] and show that $S_{0}$ and $S_{1}$ do not have to be intervals. As long as $S_{0}$ and $S_{1}$ are disjoint aliasfree $(T)$ zones [i.e., $x(t)$ is $U(T, 2)] x(t)$ can be recovered from $\operatorname{PNS}(2)$ samples. An example of $U(T, 2)$ signal $x(t)$ is shown in Fig. 12(a). We can verify that $S_{0}$ and $S_{1}$ as indicated in Fig. 12(a) are aliasfree $(T)$ and $x(t)$ is $U(T, 2)$, where $T=2 \pi / \sigma$.

In fact, we will show that reconstruction from PNS samples can be achieved for the more general $U(T, L)$ signals, those whose frequency supports can be expressed as the union of $L$ nonoverlapping aliasfree $(T)$ sets. In this case, we use PNS sampling of $L$ th order $[\operatorname{PNS}(L)]$. In the $\operatorname{PNS}(L)$ sampling of $x(t)$, there are $L$ sets of samples, $x\left(n T+d_{0}\right), x(n T+$ $\left.d_{1}\right), \cdots, x\left(n T+d_{L-1}\right)$. Referring to Fig. 7, the sampling period is $T$ in each channel, and the average sampling period is $T / L$. As the total bandwidth of $x(t)$ is $L \sigma$; aliasing occurs in each channel. We will see that judicious choice of delays $d_{\ell}$ and synthesis filters enable the reconstruction of $x(t)$ from $\operatorname{PNS}(L)$ samples.

In the $\ell$ th channel, $y_{\ell}(t)$ contains samples $x\left(n T+d_{\ell}\right)$ and $Y_{\ell}(\nu)$ consists of shifted versions of $X(\nu)$

$$
Y_{\ell}(\nu)=\frac{1}{T} \sum_{k \in \mathcal{Z}} X(\nu-k \sigma) e^{-j k d_{\ell} \sigma} .
$$

By the $U(T, L)$ nature of $X(\nu)$, we can partition the support of $X(\nu)$ into $L$ nonoverlapping aliasfree $(T)$ sets, $S_{0}, S_{1}, \cdots, S_{L-1}$. Define $X_{i}(\nu)$ to be the part of $X(\nu)$ on $S_{i}$, i.e., $X_{i}(\nu)=X(\nu) \chi_{S_{i}}(\nu)$. Each $X_{i}(\nu)$ is aliasfree $(T)$; its shifted copies fill the whole frequency line upon sampling at a period $T$.

Consider only the frequencies on the set $S_{i}$. In addition to $X_{i}(\nu)$, the signal $Y_{\ell}(\nu)$ contains $L-1$ shifted copies, one from each $X_{m}(\nu)$, for $m \neq i$. Say these shifted copies are $\left\{X_{m}(\nu)\right\}_{m \neq i}$ shifted, respectively, by $\beta_{1}(\nu) \sigma, \beta_{2}(\nu) \sigma, \cdots, \beta_{L-1}(\nu) \sigma$. Then,

$$
\begin{aligned}
T Y_{\ell}(\nu)= & X_{i}(\nu)+X_{0}\left[\nu-\beta_{1}(\nu) \sigma\right] e^{-j \beta_{1}(\nu) d_{\ell} \sigma}+\cdots \\
& +X_{i-1}\left[\nu-\beta_{i}(\nu) \sigma\right] e^{-j \beta_{i}(\nu) d_{\ell} \sigma} \\
& +X_{i+1}\left[\nu-\beta_{i+1}(\nu) \sigma\right] e^{-j \beta_{i+1}(\nu) d_{\ell} \sigma}+\cdots \\
& +X_{L-1}\left[\nu-\beta_{L-1}(\nu) \sigma\right] e^{-j \beta_{L-1}(\nu) d_{\ell} \sigma}
\end{aligned}
$$

for $\nu \in S_{i}$. Because $X_{i}(\nu)$ are nonoverlapping, $Y_{\ell}(\nu)$ can be rewritten as

$$
\begin{aligned}
& Y_{\ell}(\nu)=\frac{1}{T}\left\{X(\nu)+\sum_{i=1}^{L-1} X\left[\nu-\beta_{i}(\nu) \sigma\right] e^{-j \beta_{i}(\nu) d_{\ell} \sigma}\right\}, \\
& \quad \text { for } \nu \in \mathcal{S}(X) .
\end{aligned}
$$

Notice that the functions $\beta_{i}(\nu)$ thus defined are piecewise constant over $\mathcal{S}(X)$ because $\mathcal{S}(X)$ is the union of finitely many intervals. Under mild conditions to be discussed below, there will exist a set of reconstruction filters $\left\{F_{\ell}(\nu)\right\}$ such that that aliasing terms $X\left[\nu-\beta_{i}(\nu) \sigma\right]$ are cancelled and $\hat{X}(\nu)=X(\nu)$ in Fig. 7. The details are given next.

Lemma 3: A $U(T, L)$ signal $x(t)$ can be recovered from its $\operatorname{PNS}(L)$ samples if the equation below has a solution for every $\nu \in \mathcal{S}(X)$.

$$
\begin{gathered}
\underbrace{\left(\begin{array}{cccc}
1 & 1 & \cdots & 1 \\
e^{-j \beta_{1}(\nu) d_{0} \sigma} & e^{-j \beta_{1}(\nu) d_{1} \sigma} & \cdots & e^{-j \beta_{1}(\nu) d_{L-1} \sigma} \\
e^{-j \beta_{2}(\nu) d_{0} \sigma} & e^{-j \beta_{2}(\nu) d_{1} \sigma} & \cdots & e^{-j \beta_{2}(\nu) d_{L-1} \sigma} \\
\vdots & \vdots & \ddots & \vdots \\
e^{-j \beta_{L-1}(\nu) d_{0} \sigma} & e^{-j \beta_{L-1}(\nu) d_{1} \sigma} & \cdots & e^{-j \beta_{L-1}(\nu) d_{L-1} \sigma}
\end{array}\right)}_{A(\nu)} \\
\underbrace{\left(\begin{array}{c}
F_{0}(\nu) \\
F_{1}(\nu) \\
F_{2}(\nu) \\
\vdots \\
F_{L-1}(\nu)
\end{array}\right)}_{\boldsymbol{f}(\nu)}=T \underbrace{\left(\begin{array}{c}
1 \\
0 \\
0 \\
\vdots \\
0
\end{array}\right)}_{\boldsymbol{e}_{0}} .
\end{gathered}
$$

In particular, if the matrix $\boldsymbol{A}(\nu)$ is nonsingular, we can solve (11) and obtain the synthesis filters. 
Proof of Lemma 3: The recombined signal $\hat{X}(\nu)=\sum_{\ell=0}^{L-1}$ $Y_{\ell}(\nu) F_{\ell}(\nu)$, or

$$
\hat{X}(\nu)=\frac{1}{T} \sum_{\ell=0}^{L-1} F_{\ell}(\nu) \sum_{k \in \mathcal{Z}} X(\nu-k \sigma) e^{-j k d_{\ell} \sigma} .
$$

i) When $\nu \notin \mathcal{S}(X)$ : By the expression $\hat{X}(\nu)=$ $\sum_{\ell=0}^{L-1} Y_{\ell}(\nu) F_{\ell}(\nu)$, we have $\hat{X}(\nu)=X(\nu)=0$, if the synthesis filters $F_{\ell}(\nu)$ are zero outside of support of $X(\nu)$.

ii) When $\nu \in \mathcal{S}(X)$ : We can use (10) to simplify the expression of $\hat{X}(\nu)$,

$$
\begin{aligned}
& T \hat{X}(\nu) \\
& =\sum_{\ell=0}^{L-1} F_{\ell}(\nu)\left\{X(\nu)+\sum_{i=1}^{L-1} X\left[\nu-\beta_{i}(\nu) \sigma\right] e^{-j \beta_{i}(\nu) d_{\ell} \sigma}\right\}, \\
& \nu \in \mathcal{S}(X) .
\end{aligned}
$$

A rearrangement of the above expression gives us

$$
\begin{aligned}
T \hat{X}(\nu)= & X(\nu) \sum_{\ell=0}^{L-1} F_{\ell}(\nu)+\sum_{i=1}^{L-1} X\left[\nu-\beta_{i}(\nu) \sigma\right] \\
& \cdot \sum_{\ell=0}^{L-1} F_{\ell}(\nu) e^{-j \beta_{i}(\nu) d_{\ell} \sigma}, \quad \nu \in \mathcal{S}(X) .
\end{aligned}
$$

We can make the following observations. For $\nu \in \mathcal{S}(X)$, the reconstructed signal is free from aliasing error if $\sum_{\ell=0}^{L-1} F_{\ell}(\nu) e^{-j \beta_{i}(\nu) d_{\ell} \sigma}=0$, for $i=1,2, \cdots, L-1$ and in which case $\hat{X}(\nu)$ is simply $(1 / T) X(\nu) \sum_{\ell=0}^{L-1} F_{\ell}(\nu)$.

Combining i) and ii), we can reconstruct $x(t)$ successfully if the synthesis filters $F_{\ell}(\nu)$, for $\ell=0,1, \cdots, L-1$, are nonzero only on the support of $X(\nu)$ and if for $\nu \in \mathcal{S}(X)$ the synthesis filters satisfy

$$
\begin{gathered}
\sum_{\ell=0}^{L-1} F_{\ell}(\nu)=T, \quad \text { and } \sum_{\ell=0}^{L-1} F_{\ell}(\nu) e^{-j \beta_{i}(\nu) d_{\ell} \sigma}=0, \\
i=1,2, \cdots, L-1 .
\end{gathered}
$$

These conditions can be written as the matrix form in (11).

Remarks: If $A(\nu)$ is nonsingular on the support of $X(\nu)$, the synthesis filters are unique on the support of $X(\nu)$. For $\nu \notin \mathcal{S}(X)$, we can follow a procedure similar to that in Lemma 3 and show that for $\nu \notin \mathcal{S}(X)$,

$$
\hat{X}(\nu)=X(\nu)=0, \quad \text { if and only if } \boldsymbol{A}^{\prime}(\nu) \boldsymbol{f}(\nu)=\mathbf{0}
$$

where $A^{\prime}(\nu)$ is nonsingular for all $\nu \notin \mathcal{S}(X)$ whenever $A(\nu)$ is nonsingular for every frequency $\nu$ on support of $X(\nu)$. Therefore, if $\boldsymbol{A}(\nu)$ is nonsingular on the support of $X(\nu)$ [hence $A^{\prime}(\nu)$ is nonsingular for $\nu \notin \mathcal{S}(X)$ ], then the synthesis filters $F_{\ell}(\nu)$ are necessarily zero for $\nu \notin \mathcal{S}(X)$.
Notice that if we choose $d_{\ell}=\ell d_{1}$, for $\ell=0,1, \cdots, L-1$. Then $A(\nu)$ becomes a Vandermonde matrix as shown in (14) at the bottom of the page. The condition for nonsingularity becomes much more tractable. More precisely, we have the following theorem.

Theorem 2: Consider a $U(T, 2)$ signal $x(t)$. There always exist $\left\{d_{\ell}\right\}_{\ell=0}^{L-1}$ and synthesis filters $\left\{F_{\ell}(\nu)\right\}_{\ell=0}^{L-1}$ such that $x(t)$ can be reconstructed from $x\left(n T+d_{0}\right), x\left(n T+d_{1}\right), \cdots, x(n T+$ $\left.d_{L-1}\right)$ and $x(t)=\sum x\left(n T+d_{\ell}\right) f_{\ell}(t-n T)$. In particular, we can choose

$$
\begin{gathered}
d_{\ell}=\ell d_{1}, \quad \ell=0,1, \cdots, L-1 \\
d_{1} \beta_{i}(\nu) / T \text { and } d_{1}\left[\beta_{i}(\nu)-\beta_{m}(\nu)\right] / T, \\
i \neq m, \text { are not integers for any } \nu \in \mathcal{S}(X) .
\end{gathered}
$$

The existence of such $d_{1}$ is guaranteed. In this case, $A(\nu)$ is nonsingular and $\boldsymbol{f}(\nu)=\left[F_{0}(\nu) \cdots F_{L-1}(\nu)\right]^{T}$ is given by

$$
f(\nu)=T A(\nu)^{-1} e_{0} \chi_{\mathcal{S}(X)}(\nu)
$$

where $A(\nu)$ is as given in (14) and $\boldsymbol{e}_{0}=\left[\begin{array}{llll}1 & 0 & \cdots & 0\end{array}\right]^{T}$. Furthermore, in this case, the reconstruction is stable.

Proof: The condition for nonsingularity of the Vandermonde matrix in (14) is

$$
e^{-j \beta_{i}(\nu) d_{1} \sigma} \neq 1, \quad \text { and } e^{-j \beta_{i}(\nu) d_{1} \sigma} \neq e^{-j \beta_{m}(\nu) d_{1} \sigma}, i \neq m \text {. }
$$

This can be rewritten as (15). The nonoverlapping property among $X_{i}(\nu)$ implies that $\beta_{i}(\nu)-\beta_{m}(\nu) \neq 0$, whenever $i \neq m$. On the other hand, the support of $X(\nu)$ consists of finitely many intervals; $\beta_{i}(\nu)$ and $\beta_{i}(\nu)-\beta_{m}(\nu)$ can take on only finitely many integer values. So we can always find rational $d_{1}$ that satisfies (15). For a chosen $d_{1}$, solving (11) gives us the solutions of the synthesis filters $F_{\ell}(\nu)$, which are functions of $\beta_{i}(\nu)$. The piecewise constant property of $\beta_{i}(\nu)$ implies that $F_{\ell}(\nu)$ are also piecewise constant and can be viewed as a linear combination of some ideal brick-wall filters. Therefore, following the reasoning in Section II-B, we conclude that the reconstruction of $x(t)$ from its $\operatorname{PNS}(L)$ samples is stable.

\section{Remarks:}

1) Under the assumption that $\mathcal{S}(X)$ is the union of $L$ nonoverlapping aliasfree $(T)$ zones, $\beta_{i}(\nu)$ is unique for any frequency $\nu$. Because $S_{i}$ are now union of intervals, $\beta_{i}(\nu)$ could take on more than four values, which is the case for two-band signals. The number of intervals contained in $S_{i}$ is finite and so is the number of values $\beta_{i}(\nu)$ can assume.

2) We only address the class of signals whose supports are the union of $L$ nonoverlapping aliasfree $(T)$ zones. In this case, the signals have total bandwidth $L \sigma$. For a signal $X(\nu)$ whose support is the union of $L$ overlapping

$$
A(\nu)=\left[\begin{array}{ccccc}
1 & 1 & 1 & \cdots & 1 \\
1 & e^{-j \beta_{1}(\nu) d_{1} \sigma} & e^{-j 2 \beta_{1}(\nu) d_{1} \sigma} & \cdots & e^{-j(L-1) \beta_{1}(\nu) d_{1} \sigma} \\
1 & e^{-j \beta_{2}(\nu) d_{1} \sigma} & e^{-j 2 \beta_{2}(\nu) d_{1} \sigma} & \cdots & e^{-j(L-1) \beta_{2}(\nu) d_{1} \sigma} \\
\vdots & \vdots & & \ddots & \vdots \\
1 & e^{-j \beta_{L-1}(\nu) d_{1} \sigma} & e^{-j 2 \beta_{L-1}(\nu) d_{1} \sigma} & \cdots & e^{-j(L-1) \beta_{L-1}(\nu) d_{1} \sigma}
\end{array}\right]
$$




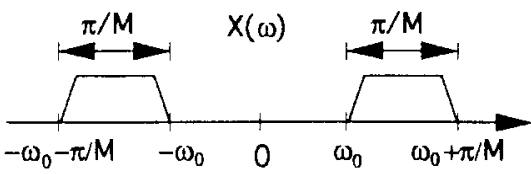

Fig. 13. Band-limited aliasfree $(M)$ signal with total bandwidth $2 \pi / M$.

aliasfree $(T)$ zones, the actual total bandwidth could be $\leq L \sigma$. In this case, we can always add to $\mathcal{S}(X)$ some hypothetical region to make it the union of $L$ nonoverlapping aliasfree $(T)$ zones, and the above theorem can be applied.

3) Stability of Reconstruction. The synthesis filters $F_{\ell}(\nu)$ are nonzero only on $\mathcal{S}(X)$ according to (7). On $\mathcal{S}(X)$ they are functions of $\beta_{i}(\nu)$ and are hence piecewise constant. Much like the two-band case in the previous section, the synthesis filters $F_{\ell}(\nu)$ can be regarded as a linear combination of some ideal brick-wall filters; the argument for stability of reconstruction in two-band case continues to hold for the $U(T, L)$ class.

Example 1: Consider the $U(T, 2)$ signal $X(\nu)$ shown in Fig. 12(a). For $L=2$, there is only one beta function $\beta(\nu)$. The shifted versions of $X_{1}(\nu)$ are as shown in Fig. 12(b), which shows that $X_{1}(\nu-3 \sigma)$ and $X_{1}(\nu-4 \sigma)$ overlap with $X_{0}(\nu)$. In the interval where $X_{1}(\nu-3 \sigma)$ overlaps with $X_{0}(\nu)$, the value of $\beta(\nu)$ is 3 and in the interval where $X_{1}(\nu-4 \sigma)$ overlaps with $X_{0}(\nu)$, the value of $\beta(\nu)$ is 4 . A sketch of $\beta(\nu)$ is given in Fig. 12(c). In this case, $\beta(\nu)$ is a piecewise constant function with constant values in six intervals. For successful reconstruction of $x(t)$ from $x(T n)$ and $x\left(T n+d_{1}\right)$, the value of $d_{1}$ should be such that $d_{1} \beta(\nu) / T$ is not an integer for any $\nu \in \mathcal{S}(X)$. As $\beta(\nu)$ assumes four values \pm 3 and $\pm 4, d_{1}$ can be any real number other than $n T / 3$ and $n T / 4$, where $n$ is any integer. For example, $d_{1}$ can be any number in the interval $(0, T / 4)$. The synthesis filter $F_{0}(\nu)$, for the choice $d_{1}=T / 5$, is as sketched in Fig. 12(d). The other synthesis filter $F_{1}(\nu)=F_{0}(-\nu)$.

Discrete Time Case: If a discrete time signal $x(n)$ has Fourier transform $X(\omega)$ restricted to the intervals shown in Fig. 13, we can decimate $x(n)$ by $M$ without creating aliasing. This idea is routinely used in cosine modulated filter banks [19]. But if the bands are not located at integer multiple of $\pi / M$, we need to use periodically nonuniform sampling techniques. In the discrete time case, decimation of a signal by an integer $M$ is equivalent to retaining the first polyphase component of that signal; in a periodically nonuniform sampling of $L$ th order (Fig. 8), the $L$ sets of samples are the $d_{0}$ th, $d_{1}$ th, $\cdots, d_{L-1}$ th polyphase components. Similar to the continuous time case, aliasfree $(M)$ property for discrete time sequences and aliasfree $(M)$ zones can be defined. We use $U(M, L)$ to denote the class of signals whose frequency supports are the union of $L$ nonoverlapping aliasfree $(M)$ zones. Note that in Fig. 8, the total data is $L / M$ times the original input; the nonuniform sampling scheme makes sense only for $L<M$. In the 1-D continuous time case, we saw that the class $U(T, L)$ allows reconstruction from $\operatorname{PNS}(L)$ samples. Generalizations to the 1-D discrete time case follow fairly routinely. However, such generalizations fail in

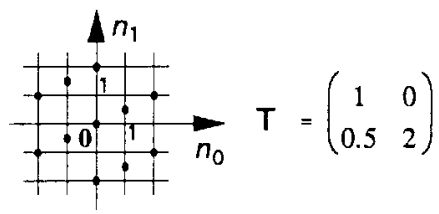

Fig. 14. Lattice of $T$.

the 2-D discrete time case, which will be discussed in Section $\mathrm{V}$.

\section{Two-Dimensional Continuous Time Uniforn AND PERIODICALly NONUNIFORM SAMPLING}

For 2-D signals, aliasfree $(T)$ property and aliasfree $(T)$ zones can be defined as in the one-dimensional (1-D) case. But now the sampling period is a $2 \times 2$ nonsingular matrix $\boldsymbol{T}$ and the samples are located on the lattice defined by $\boldsymbol{T}$, i.e., located at $\boldsymbol{T} \boldsymbol{n}$ for all integer vectors $\boldsymbol{n}$. For example, the lattice of

$$
\boldsymbol{T}=\left[\begin{array}{cc}
1 & 0 \\
0.5 & 2
\end{array}\right]
$$

is as shown in Fig. 14. A 2-D signal $x(\boldsymbol{t})$ is called aliasfree $(\boldsymbol{T})$ if the sampling of $x(\boldsymbol{t})$ with matrix $T$ does not create aliasing. In this case, the support of $X(\boldsymbol{\nu})$ [Fourier transform of $x(\boldsymbol{t})$ ] is called an aliasfree $(\boldsymbol{T})$ zone or aliasfree $(\boldsymbol{T})$ set.

As mentioned in Section I, the sampling theorem for twoband signals is well known: a two-band signal $x(t)$ with total bandwidth $\Theta$ (Fig. 4) allows reconstruction from uniform samples $x[(2 \pi / \Theta) n]$ if band edge $\nu_{0}$ is a integer multiple of $\Theta / 2$. A two-dimensional (2-D) extension of two-band signals is the class of two-parallelogram (Two-P) signals [18]. A signal $X(\boldsymbol{\nu})$ is called Two-P if its support consists of two identical parallelograms, e.g., as shown in Fig. 15. We derive in Section IV-A a sampling theorem for the Two-P class parallel to that for 1-D two-band signals. When reconstruction from uniform samples fails, we then seek reconstruction from periodically nonuniform samples, analogous to Section III. In Section IV-B, we present a periodically nonuniform sampling theorem for the more general class $U(\boldsymbol{T}, L)$, which is the collection of signals whose frequency supports are the union of $L$ aliasfree $(\boldsymbol{T})$ sets. The Two-P class is, by definition, a subclass of $U(T, 2)$.

\section{A. Sampling Theorem for Continuous Time Two-P Signals}

Recall that the key issue in the 1-D bandpass sampling theorem is to sample at the critical sampling rate without creating aliasing, so that we can reconstruct the original signal from samples. The sampling rate represents how fast the samples are acquired or how densely located the samples are. The second meaning in the 2-D case is represented by the quantity called sampling density. The sampling density $\rho$ for a sampling matrix $\boldsymbol{T}$ is

$$
\rho=\frac{1}{|\boldsymbol{T}|} .
$$

Consider a Two- $P$ signal $x(\boldsymbol{t})$ (as shown in Fig. 15) whose two parallelograms are shifted versions of $S P D\left(\pi \boldsymbol{N}^{-T}\right)$, 


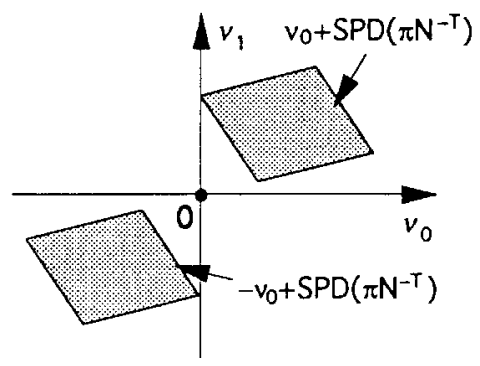

Fig. 15. Typical support of a two-parallelogram signal.

where the symmetric parallelepiped $S P D(\boldsymbol{V})$ of a matrix $V$ is the set

$$
S P D(V)=\left\{\boldsymbol{V} \boldsymbol{x}, \boldsymbol{x} \in[-1,1)^{2}\right\} .
$$

For a one-parallelogram signal with frequency support $S P D\left(\pi N^{-T}\right)$, the critical sampling density is $1 /|\boldsymbol{N}|$. The area of the support of $X(\nu)$ is twice that of $\operatorname{SPD}\left(\pi \boldsymbol{N}^{-T}\right)$; the critical sampling density for $X(\nu)$ is $2 /|N|$. So the sampling theorem to be established for the Two-P class is a necessary and sufficient condition such that $x(\boldsymbol{t})$ allows critical alias free sampling, i.e., $x(\boldsymbol{t})$ can be reconstructed from $x(\boldsymbol{T t})$, where $\boldsymbol{T}$ is some $2 \times 2$ matrix with $|\boldsymbol{T}|=|\boldsymbol{N}| / 2$. The 1-D sampling theorem for two-band signals hints that the two parallelograms in the support of $X(\boldsymbol{\nu})$ should be somehow properly located. The details of this are given in the following theorem.

Theorem 3: Let $x(\boldsymbol{t})$ be a continuous time Two-P signal and let the support of $X(\boldsymbol{\nu})$ be the union of two parallelograms described by $\nu_{0}+S P D\left(\pi \boldsymbol{N}^{-T}\right)$ and $-\nu_{0}+S P D\left(\pi \boldsymbol{N}^{-T}\right)$. Define $r_{0}=N^{T} \nu_{0} / \pi$. Then $x(\boldsymbol{t})$ is aliasfree $(T)$ for some matrix $T$ satisfying $|\boldsymbol{T}|=|\boldsymbol{N}| / 2$ if and only if the following is true: the vector $\boldsymbol{r}_{0}$ has at least one nonzero integer element.

Proof of Theorem 3: (Necessity of the condition.) Recall that when we sample a signal $x(\boldsymbol{t})$ using a sampling matrix $\boldsymbol{T}$, the Fourier transform of the output is

$$
\frac{1}{|\boldsymbol{T}|} \sum_{\boldsymbol{k} \in \mathcal{Z}^{2}} \hat{X}_{\boldsymbol{k}}(\boldsymbol{\nu}), \quad \text { where } \hat{X}_{\boldsymbol{k}}(\boldsymbol{\nu})=X\left(\boldsymbol{T}^{-T} \boldsymbol{\nu}-2 \pi \boldsymbol{T}^{-T} \boldsymbol{k}\right)
$$

which consists of shifted and expanded versions of $X(\boldsymbol{\nu})$. The expanded version $\hat{X}_{\boldsymbol{k}}(\boldsymbol{\nu})$ consists of two identical parallelograms that are shifted versions of $\operatorname{SPD}\left(\pi L^{-T}\right)$, where $\boldsymbol{L}=\boldsymbol{T}^{-1} \boldsymbol{N}$. If $|\boldsymbol{T}|=|N| / 2$, the frequency plane will be filled by $\hat{X}_{\boldsymbol{k}}(\boldsymbol{\nu})$. So if $x(\boldsymbol{t})$ is aliasfree $(\boldsymbol{T})$, the frequency plane is tiled by the parallelogram of $S P D\left(\pi L^{-T}\right)$.

For convenience, we normalize the frequency plane by $2 \pi L^{-T}$; the new axes $\mu_{0}$ and $\mu_{1}$ are the two entries of $\boldsymbol{\mu}=$ $2 \pi \boldsymbol{L}^{-T} \boldsymbol{\nu}$. After normalization, the support of $\hat{X}_{\boldsymbol{k}}(\boldsymbol{\mu})$ appears as the union of two squares (Fig. 16), denoted by $S$ and $S^{\prime}$, with $S=-\boldsymbol{r}_{0} / 2+S P D(0.5 \boldsymbol{I})$ and $S^{\prime}=\boldsymbol{r}_{0} / 2+S P D(0.5 I)$ and the relative position of $S$ and $S^{\prime}$ is described by the vector $\boldsymbol{r}_{0}$. So if the original frequency plane is tiled by the parallelogram of $S P D\left(\pi L^{-T}\right)$, the new normalized plane is tiled by the unit squares of $S P D(0.5 I)$. Observe that in a square tiling, we can always find at least one set of parallel lines (Fig. 17) and all the cells are bounded by these lines. For example, in the tiling of Fig. 17(a), we can observe one set of parallel lines and all the squares are bounded by the horizontal

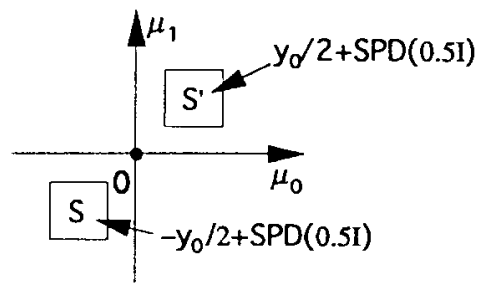

Fig. 16. Support of a two-parallelogram signal $X(\nu)$ with normalized axes.

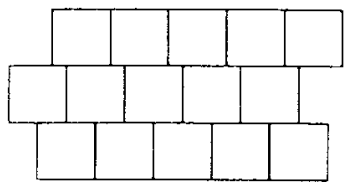

(a)

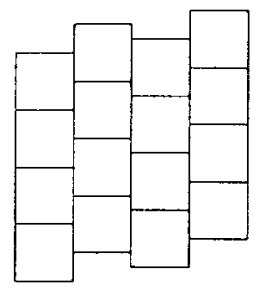

(b)

Fig. 17. Square tiling with (a) horizontal lines and (b) vertical lines.

lines (horizontal square tiling). In the tiling of Fig. 17(b), however, we can observe vertical lines, and the squares are bounded by these vertical lines (vertical square tiling). Notice that in a horizontal tiling, any two unit squares have integer vertical distance, whereas in a vertical tiling, any two cells have integer horizontal distance. So the passbands $S$ and $S^{\prime}$, being two cells in a horizontal or vertical tiling, have integer horizontal or vertical distance. As $S$ and $S^{\prime}$ are separated by $\boldsymbol{r}_{0}$, the vector $\boldsymbol{r}_{0}$ must have one integer element. When $\boldsymbol{r}_{0}$ has one zero element, say $\left[\boldsymbol{r}_{0}\right]_{0}=0$, the squares $S$ and $S^{\prime}$ are confined to the same two vertical parallel lines-vertical tiling. So the vertical distance between $S$ and $S^{\prime}$ is necessarily an integer as well, i.e., $\left[\boldsymbol{r}_{0}\right]_{1}$ is also an integer. Therefore $\boldsymbol{r}_{0}$ must have at least one nonzero element.

Sufficiency of the Condition: To show the condition is sufficient, we will construct a sampling matrix $\boldsymbol{T}=N L^{-1}$ with $|\boldsymbol{L}|=2$ such that $x(\boldsymbol{t})$ is aliasfree $(\boldsymbol{T})$. In particular, the following $L$ can be used for i) when $\left[\boldsymbol{r}_{0}\right]_{0}$ is a nonzero integer and ii) when $\left[\boldsymbol{r}_{0}\right]_{1}$ is a nonzero integer:

$$
\begin{aligned}
\boldsymbol{L} & =\left[\begin{array}{cc}
1 & \left(\left[\boldsymbol{r}_{0}\right]_{1}-1\right) /\left[\boldsymbol{r}_{0}\right]_{0} \\
0 & 2
\end{array}\right] \\
\boldsymbol{L} & =\left[\begin{array}{cc}
2 & 0 \\
\left(\left[\boldsymbol{r}_{0}\right]_{0}-1\right) /\left[\boldsymbol{r}_{0}\right]_{1} & 1
\end{array}\right] .
\end{aligned}
$$

It can be verified that corresponding to these two choices, shifts of $S$ constitute the patterns in Fig. 18(a) and (b). It can be further verified that the blank space left will be filled by the shifts of $S^{\prime}$ when $\boldsymbol{L}$ is given above. That is, the shifts of $S$ and $S^{\prime}$ are interlaced perfectly; and $x(\boldsymbol{t})$ is aliasfree $(\boldsymbol{T})$.

Remark: The preceding theorem shows that the relative positions of the two parallelograms determines whether a Two- 


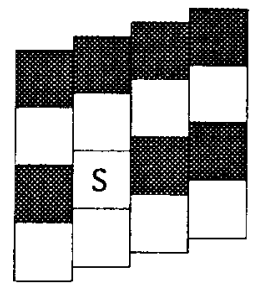

(a)

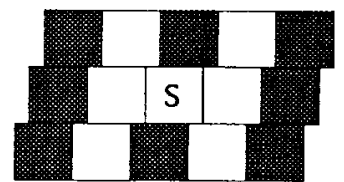

(b)

Fig. 18. The patterns formed by $S$ and its shifts corresponding to the two cases of $L$ in (15).

$P$ signal $x(\boldsymbol{t})$ can be recovered from $x(\boldsymbol{T t})$ for some matrix $\boldsymbol{T}$ with $|\boldsymbol{T}|=|N| / 2$. However, for a given matrix $\boldsymbol{T}$ with $|T|=|\boldsymbol{N}| / 2$, whether $x(\boldsymbol{t})$ is aliasfree $(T)$ depends not only on the relative positions of two parallelograms, but also on the shape of the parallelograms. In particular, it can be shown that $\boldsymbol{L}=\boldsymbol{T}^{-1} \boldsymbol{N}$ must have one integer column vector. This situation does not arise in the previous 1-D case because in 1-D shapes are not involved.

\section{B. Periodically Nonuniform Sampling and Reconstruction}

In this subsection we will show that 2-D $U(\boldsymbol{T}, L)$ signals can be recovered from samples obtained through $L$ th-order periodically nonuniform sampling, i.e., samples at $\left\{\boldsymbol{T} \boldsymbol{n}+\boldsymbol{d}_{0}\right\},\{\boldsymbol{T} \boldsymbol{n}+$ $\left.\boldsymbol{d}_{1}\right\}, \cdots,\left\{\boldsymbol{T} \boldsymbol{n}+\boldsymbol{d}_{L-1}\right\}$. A sampling and reconstruction theorem similar to that derived in the previous section for 1-D $U(T, L)$ signals will be developed.

In each channel, $y_{\ell}(\boldsymbol{t})$ contains samples $x\left(\boldsymbol{n} \boldsymbol{T}+\boldsymbol{d}_{\ell}\right)$, and $Y_{\ell}(\nu)$ consists of shifted versions of $X(\nu)$,

$$
Y_{\ell}(\boldsymbol{\nu})=\frac{1}{|\boldsymbol{T}|} \sum_{\boldsymbol{k} \in \mathcal{Z}^{2}} X\left(\boldsymbol{\nu}-2 \pi \boldsymbol{T}^{-T} \boldsymbol{k}\right) e^{-j 2 \pi \boldsymbol{k}^{T} \boldsymbol{T}^{-1} \boldsymbol{d}_{\ell}}
$$

Since $x(\boldsymbol{t})$ is $U(\boldsymbol{T}, L)$, similar to the 1-D case, $Y_{\ell}(\boldsymbol{\nu})$ can be written as the sum of $X(\nu)$ and $L-1$ shifted copies of $X(\boldsymbol{\nu})$. Denoting these shifted copies by $X\left[\boldsymbol{\nu}-\beta_{i}(\boldsymbol{\nu}) \sigma\right]$, for $i=1,2, \cdots, L-1$, we have (17), shown at the bottom of the page. By imitating the procedures in the proof of Lemma 3, the following lemma can be shown.

Lemma 4: A 2-D $U(\boldsymbol{T}, L)$ signal $x(\boldsymbol{t})$ can be reconstructed from $x\left(\boldsymbol{T} n+\boldsymbol{d}_{0}\right), x\left(\boldsymbol{T} \boldsymbol{n}+\boldsymbol{d}_{1}\right), \cdots, x\left(\boldsymbol{T} n+\boldsymbol{d}_{L-1}\right)$ if and only if the equation below has a solution for every $\boldsymbol{\nu} \in \mathcal{S}(X)$.

$$
\boldsymbol{A}(\boldsymbol{\nu})\left[\begin{array}{lllll}
F_{0}(\boldsymbol{\nu}) & F_{1}(\boldsymbol{\nu}) & \cdots & F_{L-1}(\boldsymbol{\nu})
\end{array}\right]^{T}=\left[\begin{array}{lllll}
T & 0 & 0 & \cdots & 0
\end{array}\right]^{T}
$$

where the $L \times L$ matrix $A(\boldsymbol{\nu})$ is given by (19), shown at the bottom of the page. If $A(\nu)$ is nonsingular for all $\nu \in \mathcal{S}(X)$, we can solve (18) and obtain the synthesis filters.

Observe that the choice

$$
d_{\ell}=\ell d_{1}, \quad \ell=0,1, \cdots, L-1
$$

leads to a Vandermonde $\boldsymbol{A}(\boldsymbol{\nu})$ for all $\boldsymbol{\nu} \in \mathcal{S}(X)$. If, furthermore, there exists $\boldsymbol{d}_{1}$ such that $\boldsymbol{A}(\boldsymbol{\nu})$ is nonsingular, then we can always reconstruct $U(T, L)$ signals from PNS samples. The existence of such $d_{1}$ is guaranteed as to be shown in the theorem to follow.

Theorem 4: A 2-D $U(T, L)$ signal $x(\boldsymbol{t})$ can be reconstructed from PNS samples $x\left(\boldsymbol{T n}+\boldsymbol{d}_{0}\right), x(T n+$ $\left.\boldsymbol{d}_{1}\right), \cdots, x\left(\boldsymbol{T} \boldsymbol{n}+\boldsymbol{d}_{L-1}\right)$.

Proof: This will be done in two steps. We first show that nonsingularity of $\boldsymbol{A}(\boldsymbol{\nu})$ is assured if $\boldsymbol{d}_{1}$ is such that

$$
\beta_{i}^{T}(\nu) T^{-1} d_{1} \neq \text { integer }
$$

and

$$
\left[\boldsymbol{\beta}_{i}^{T}(\boldsymbol{\nu})-\boldsymbol{\beta}_{m}^{T}(\boldsymbol{\nu})\right] T^{-1} \boldsymbol{d}_{1} \neq \text { integer, } \text { for } i \neq m .
$$

Then we show that there always exists such $d_{1}$.

i) The matrix $\boldsymbol{A}(\boldsymbol{\nu})$ is nonsingular if

$$
\begin{aligned}
& \quad e^{-j 2 \pi \boldsymbol{\beta}_{i}^{T}(\boldsymbol{\nu}) \boldsymbol{T}^{-1} \boldsymbol{d}_{1}} \neq 1 \\
& \text { and } \\
& \qquad e^{-j 2 \pi \boldsymbol{\beta}_{i}^{T}(\boldsymbol{\nu}) \boldsymbol{T}^{-1} \boldsymbol{d}_{1}} \neq e^{-j 2 \pi \boldsymbol{\beta}_{m}^{T}(\boldsymbol{\nu}) \boldsymbol{T}^{-1} \boldsymbol{d}_{1}}, \quad \text { for } i \neq m .
\end{aligned}
$$

This condition can be rewritten as (20).

ii) Because $\beta_{i}(\boldsymbol{\nu})$ assumes values from a finite collection of integer vectors, the total number of distinct vectors represented by $\boldsymbol{\beta}_{i}^{T}(\boldsymbol{\nu}) \boldsymbol{T}^{-1}$, for $i=1, \cdots, L-1$ and $\left[\boldsymbol{\beta}_{i}^{T}(\nu)-\boldsymbol{\beta}_{m}^{T}(\nu)\right] \boldsymbol{T}^{-1}$,

$$
Y_{\ell}(\boldsymbol{\nu})=\frac{1}{|\boldsymbol{T}|}\left\{X(\boldsymbol{\nu})+\sum_{i=1}^{L-1} X\left[\boldsymbol{\nu}-2 \pi \boldsymbol{T}^{-T} \boldsymbol{\beta}_{i}(\boldsymbol{\nu})\right] e^{-j 2 \pi \boldsymbol{\beta}_{i}(\boldsymbol{\nu})^{T} \boldsymbol{T}^{-1} \boldsymbol{d}_{\ell}}\right\}, \quad \text { for } \boldsymbol{\nu} \in \mathcal{S}(X) .
$$

$$
\boldsymbol{A}(\boldsymbol{\nu})=\left[\begin{array}{cccc}
1 & 1 & \cdots & 1 \\
e^{-j 2 \pi \boldsymbol{\beta}_{1}^{T}(\boldsymbol{\nu}) \boldsymbol{T}^{-1} \boldsymbol{d}_{0}} & e^{-j 2 \pi \boldsymbol{\beta}_{1}^{T}(\boldsymbol{\nu}) \boldsymbol{T}^{-1} \boldsymbol{d}_{1}} & \cdots & e^{-j 2 \pi \boldsymbol{\beta}_{1}^{T}(\boldsymbol{\nu}) \boldsymbol{T}^{-1} \boldsymbol{d}_{L-1}} \\
e^{-j 2 \pi \boldsymbol{\beta}_{2}^{T}(\boldsymbol{\nu}) \boldsymbol{T}^{-1} \boldsymbol{d}_{0}} & e^{-j 2 \pi \boldsymbol{\beta}_{2}^{T}(\boldsymbol{\nu}) \boldsymbol{T}^{-1} \boldsymbol{d}_{1}} & \cdots & e^{-j 2 \pi \boldsymbol{\beta}_{2}^{T}(\boldsymbol{\nu}) T^{-1} \boldsymbol{d}_{L-1}} \\
\vdots & \vdots & \ddots & \vdots \\
e^{-j 2 \pi \boldsymbol{\beta}_{L-1}^{T}(\boldsymbol{\nu}) \boldsymbol{T}^{-1} \boldsymbol{d}_{0}} & e^{-j 2 \pi \boldsymbol{\beta}_{L-1}^{T}(\boldsymbol{\nu}) \boldsymbol{T}^{-1} \boldsymbol{d}_{1}} & \cdots & e^{-j 2 \pi \boldsymbol{\beta}_{L-1}^{T}(\boldsymbol{\nu}) \boldsymbol{T}^{-1} \boldsymbol{d}_{L-1}}
\end{array}\right]
$$




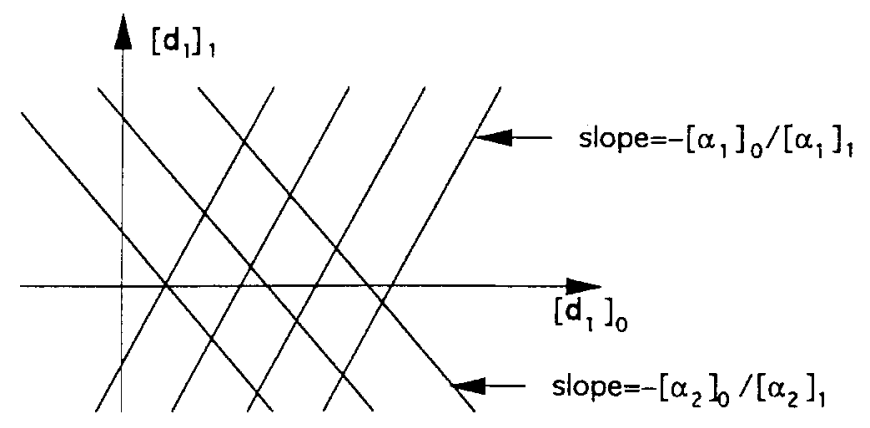

Fig. 19. An illustration pertaining to the proof of Theorem 5 .

for $i \neq m$ is finite, say $N$. Let us call these distinct $N$ vectors $\boldsymbol{\alpha}_{i}, i=1,2, \cdots, N$. The conditions in (20) can be rewritten as

$$
\left[\boldsymbol{\alpha}_{i}\right]_{0}\left[\boldsymbol{d}_{1}\right]_{0}+\left[\boldsymbol{\alpha}_{i}\right]_{1}\left[\boldsymbol{d}_{1}\right]_{1} \neq \text { integer, } \quad \text { for } i=1,2, \cdots, N
$$

If we draw a graph with $\left[\boldsymbol{d}_{1}\right]_{0}$ and $\left[\boldsymbol{d}_{1}\right]_{1}$ as the two axes, for each $i$ the equation $\left[\boldsymbol{\alpha}_{i}\right]_{0}\left[\boldsymbol{d}_{1}\right]_{0}+\left[\boldsymbol{\alpha}_{i}\right]_{1}\left[\boldsymbol{d}_{1}\right]_{1}=$ integer represents a set of parallel lines. Equation (21) says that the points on these lines are not permitted. We have $N$ sets of such parallel lines. For example, let $N=2$. Then there are two sets of parallel lines (Fig. 19) and only the points on the lines are not permitted. We can, therefore, always find $\boldsymbol{d}_{1}$ that is not on these lines, i.e., satisfies (20).

With a nonsingular $\boldsymbol{A}(\boldsymbol{\nu})$, the synthesis filters can be uniquely determined from (18).

\section{Two-Dimensional Discrete TIME SAMPLING AND RECONSTRUCTION}

In the 2-D discrete time case, aliasfree $(M)$ property, aliasfree $(\boldsymbol{M})$ zone and $U(\boldsymbol{M}, L)$ can be defined in the same manner, where $\boldsymbol{M}$ is now a $2 \times 2$ nonsingular integer matrix. In Section IV, we developed the sampling theorem for continuous time Two-P signals (Section IV-A). The discrete time counterpart of this theorem can be found in [19]. In this section, we consider the reconstruction of 2-D discrete time $U(\boldsymbol{M}, L)$ class from $P N S(L)$ samples. In the 1-D case, the discrete time results completely parallel that in the continuous time. However, the situation is quite different in the 2-D case. A 2-D discrete time $U(\boldsymbol{M}, L)$ signal $x(\boldsymbol{n})$ cannot always be reconstructed from $L$ of its polyphase components. An example of such $x(\boldsymbol{n})$ will be presented.

Following similar procedures as in previous sections, the following lemma for reconstructing $U(\boldsymbol{M}, L)$ signals can be established.

Lemma 5: A 2-D discrete time $U(\boldsymbol{M}, L)$ signal $x(\boldsymbol{n})$ can be recovered from $L$ of its polyphase components if and only if the following equation has a solution for every $\omega \in \mathcal{S}(X)$ as shown (22), shown at the bottom of the page.

In the 1-D case, we can always choose $d_{\ell}$ such that $A(\omega)$ is a nonsingular Vandermonde matrix for every $\omega \in \mathcal{S}(X)$. However, it is not always possible to do so in the 2-D case. In fact, the above equation may not have a solution for some $\boldsymbol{\omega} \in \mathcal{S}(X)$, in which case $x(\boldsymbol{n})$ cannot be reconstructed from $L$ of its polyphase components. To explain this, we take a closer look at $A(\omega)$.

The matrices $A(\omega)$ and $W^{(g)}$ : It can be verified that the matrix $A(\omega)$ above is an $L \times L$ submatrix of a matrix $W^{(g)}$, called the generalized DFT matrix, possibly with some row and column exchanges. The matrix $W^{(g)}$ is of dimensions $|\boldsymbol{M}| \times|\boldsymbol{M}|$ and the elements of $\boldsymbol{W}^{(g)}$ are given by

$$
\left[W^{(g)}\right]_{\text {in }}=e^{-j 2 \pi \boldsymbol{k}_{i}^{T} \boldsymbol{M}^{-1} \boldsymbol{m}_{n}}, \quad \boldsymbol{m}_{n} \in \mathcal{N}(\boldsymbol{M}), \boldsymbol{k}_{i} \in \mathcal{N}\left(\boldsymbol{M}^{T}\right)
$$

where the notation $\mathcal{N}(\boldsymbol{M})$ denotes the set of integer vectors of the form $\boldsymbol{M} \boldsymbol{x}, \boldsymbol{x} \in[01)^{2}$. Let $\Lambda$ be the Smith form of $\boldsymbol{M}$ [14],

$$
\Lambda=\left[\begin{array}{cc}
\lambda_{0} & 0 \\
0 & \lambda_{1}
\end{array}\right] .
$$

When $\boldsymbol{m}_{n}$ and $\boldsymbol{k}_{i}$ are properly ordered, it can be verified that $\boldsymbol{W}^{(g)}=W_{\lambda_{0}} \otimes \boldsymbol{W}_{\lambda_{1}}$, where $\boldsymbol{W}_{\lambda}$ denotes a $\lambda \times \lambda$ DFT matrix given by

$$
\left[W_{\lambda}\right]_{m n}=e^{-j(2 \pi / \lambda) m n}, \quad 0 \leq m, n<\lambda
$$

The notation $\otimes$ denotes the Kronecker product. The Kronecker product of two matrices $\boldsymbol{A}$ and $\boldsymbol{B}$ is defined as

$$
\underbrace{\boldsymbol{A}}_{I \times K} \otimes \underbrace{\boldsymbol{B}}_{J \times L}=\underbrace{\left[\begin{array}{ccc}
a_{0,0} B & \cdots & a_{0, K-1} \boldsymbol{B} \\
\vdots & \cdots & \vdots \\
a_{I-1,0} \boldsymbol{B} & \cdots & a_{I-1, K-1} \boldsymbol{B}
\end{array}\right]}_{I J \times K L} .
$$

Although DFT matrices are Vandermonde, $W^{(g)}$ is not Vandermonde in general and neither are its $L \times L$ submatrices obtained by retaining the first $L$ columns and some $L$ rows. The natural question to ask next is whether a particular set of $\left\{\boldsymbol{d}_{\ell}\right\}$ will make $\boldsymbol{A}(\boldsymbol{\omega})$ nonsingular for all $\boldsymbol{\omega} \in \mathcal{S}(X)$. In terms of the generalized DFT matrix $W^{(g)}$, the question can be recast as follows: can we find $L$ columns of $W^{(g)}$ such that for arbitrarily chosen $L$ rows of $W^{(g)}$, the resulting submatrix is always nonsingular? The answer is, unfortunately, no. Although for every frequency $\omega_{1} \in \mathcal{S}(X)$, there always exist $\left\{\boldsymbol{d}_{\ell}\right\}$ such that $A\left(\boldsymbol{\omega}_{1}\right)$ is nonsingular. The same $\boldsymbol{d}_{\ell}$ may

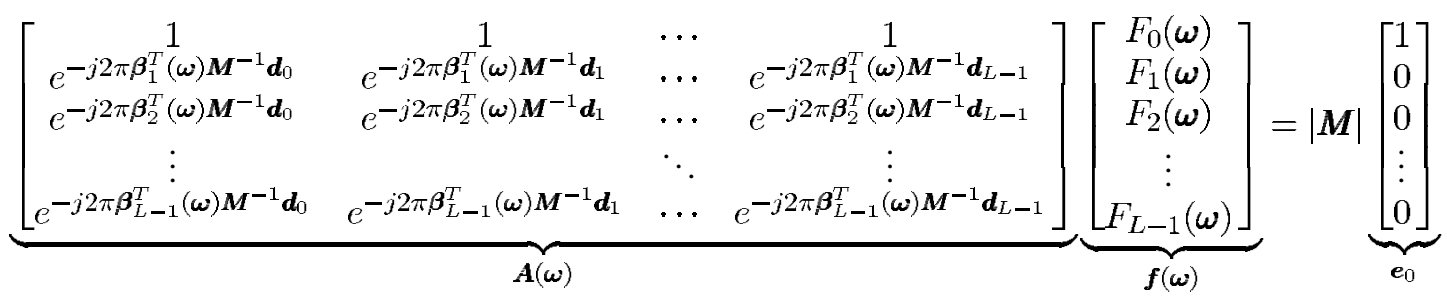


yield a singular $A\left(\omega_{1}^{\prime}\right)$ for a different frequency vector $\omega_{1}^{\prime}$. The following is an example which demonstrates that there are cases when (22) is not solvable with frequency independent $\left\{\boldsymbol{d}_{\ell}\right\}$.

Example 2: Consider a discrete time 2-D $U(\boldsymbol{M}, 2)$ signal $x(\boldsymbol{n})$, where $\boldsymbol{M}=\left[\begin{array}{l}2 \\ 0 \\ 0\end{array}\right]$ and $|\boldsymbol{M}|=4$. The four vectors in $\mathcal{N}(\boldsymbol{M})$ are

$\boldsymbol{m}_{0}=\left[\begin{array}{ll}0 & 0\end{array}\right]^{T}, \quad \boldsymbol{m}_{1}=\left[\begin{array}{ll}1 & 0\end{array}\right]^{T}, \quad \boldsymbol{m}_{2}=\left[\begin{array}{ll}0 & 1\end{array}\right]^{T}, \quad \boldsymbol{m}_{3}=\left[\begin{array}{ll}1 & 1\end{array}\right]^{T}$.

Order $\boldsymbol{k}_{i} \in \mathcal{N}\left(\boldsymbol{M}^{T}\right)$ by letting $\boldsymbol{k}_{i}=\boldsymbol{m}_{i}$, then the generalized DFT matrix $W^{(g)}$ is

$$
W^{(g)}=\left[\begin{array}{rrrr}
1 & 1 & 1 & 1 \\
1 & -1 & 1 & -1 \\
1 & 1 & -1 & -1 \\
1 & -1 & -1 & 1
\end{array}\right] \text {. }
$$

The support of $X(\boldsymbol{\omega})$, as shown in Fig. 20, consists of two aliasfree $(\boldsymbol{M})$ zones, $S_{0}$ and $S_{1}$. The set $S_{1}$ is the union of three regions $R_{0}, R_{1}$, and $R_{2}$. With $L=2$, we only have one beta function, $\beta(\omega)$. Observe that

$$
\beta(\omega)= \begin{cases}k_{1}, & \omega \in R_{0} \\ k_{2}, & \omega \in R_{1} \\ k_{3}, & \omega \in R_{2} .\end{cases}
$$

So for $\omega \in R_{0}, A(\omega)$ is a submatrix of $W^{(g)}$ obtained by keeping the zeroth and first rows of $W^{(g)}$ and two columns $W^{(g)}$. That is, $A(\omega)$ is a $2 \times 2$ sub-matrix of

$$
\left[\begin{array}{rrrr}
1 & 1 & 1 & 1 \\
1 & -1 & 1 & -1
\end{array}\right]
$$

obtained by keeping two columns. As to which two columns, it depends on the choice of $\boldsymbol{d}_{0}$ and $\boldsymbol{d}_{1}$. Without loss of generality, we can assume $\boldsymbol{d}_{0}=0$. We see that (22) has a solution for $\boldsymbol{\omega} \in R_{0}$ only if $\boldsymbol{d}_{1}$ is $\boldsymbol{m}_{1}$ or $\boldsymbol{m}_{3}$. We can do the same thing for $R_{1}$ and $R_{2}$, and reach the following necessary condition such that (22) has a solution in each $R_{i}$ :

$$
\boldsymbol{d}_{1}= \begin{cases}\boldsymbol{m}_{1} \text { or } m_{3}, & \omega \in R_{0} \\ m_{2} \text { or } m_{3}, & \omega \in R_{1} \\ m_{1} \text { or } m_{2}, & \omega \in R_{2}\end{cases}
$$

There is no common solution of $\boldsymbol{d}_{1}$ for the three regions; (22) does not have a solution for all $\boldsymbol{\omega}$ in the support of $X(\boldsymbol{\omega})$. Therefore, $x(\boldsymbol{n})$ cannot be reconstructed from two of its polyphase components.

Although it is not always possible to reconstruct a $U(\boldsymbol{M}, L)$ signal from $L$ of its polyphase components, it is always possible to do so when $\boldsymbol{M}$ assumes the following Smith form decomposition:

$$
\boldsymbol{M}=\boldsymbol{U} \Lambda \boldsymbol{V}, \quad \text { where } \Lambda=\left[\begin{array}{cc}
1 & 0 \\
0 & |\boldsymbol{M}|
\end{array}\right]
$$

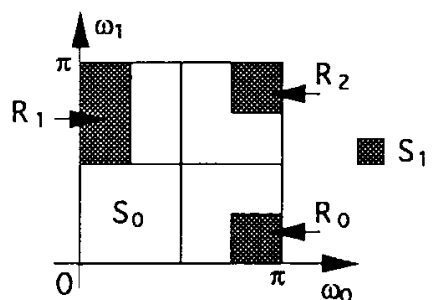

Fig. 20. A $U(\boldsymbol{M}, 2)$ signal that cannot be reconstructed from two of its polyphase components.

The corresponding generalized DFT matrix $W^{(g)}$ is the $|M| \times$ $|\boldsymbol{M}|$ DFT matrix $\boldsymbol{W}_{|\boldsymbol{M}|}$. Similar to the reconstruction of 1-D $U(M, L)$ signals, choose

$$
\boldsymbol{d}_{\ell}=\boldsymbol{U}\left[\begin{array}{l}
0 \\
1
\end{array}\right]
$$

where $\boldsymbol{U}$ is the unimodular matrix in the Smith form decomposition of $\boldsymbol{M}$ in (25). Then $\boldsymbol{A}(\boldsymbol{\omega})$ will be nonsingular for all $\boldsymbol{\omega} \in \mathcal{S}(X)$, and by (22) we can invert $A(\boldsymbol{\omega})$ to obtain the synthesis filters.

\section{CONCLUSIONS}

In this paper, we consider the reconstruction of a class of continuous time bandpass signals, the $U(T, L)$ class. The frequency supports of this class of signals consist of $L$ aliasfree $(T)$ sets. We show that $U(T, L)$ signals allow reconstruction from periodically nonuniform samples of $L$ order $[\operatorname{PNS}(L)]$. This is an extension of the work by Kohlenberg that addresses the reconstruction of two-band signals from PNS(2) samples. We have also generalized the results to the 2-D continuous time $U(\boldsymbol{T}, L)$ class and 1-D discrete time $U(M, L)$ class. In the discrete time case, the $\operatorname{PNS}(L)$ samples are essentially $L$ polyphase components of the signals. However, the generalization fails in the 2-D discrete time $U(\boldsymbol{M}, L)$; a 2-D discrete time $U(\boldsymbol{M}, L)$ signal does not allow reconstruction from $L$ polyphase components.

\section{REFERENCES}

[1] A. V. Oppenheim, A. S. Willsky, and I. Young, Signals and Systems. Englewood Cliffs, NJ: Prentice-Hall, 1983.

[2] M. M. Dodson and A. M. Silva, "Fourier analysis and the sampling theorem," IEEE Proc. Royal Irish Acad., vol. 85 A, no. 1, pp. 81-108, 1985.

[3] J. R. Higgins, "Some gap sampling series for multiband signals," Signal Process., vol. 12, no. 3, pp. 1313-1319, 1987.

[4] V. Sathe and P. P. Vaidyanathan, "Effects of multirate systems on the statistical properties of random inputs," IEEE Trans. Signal Processing, vol. 41, pp. 131-146, 1993

[5] T. Chen and P. P. Vaidyanathan, "Recent developments in multidimensional multirate systems," IEEE Trans. Circuits Syst. Video Technol., vol. 3, pp. 116-137, Apr. 1993.

[6] A. Kohlenberg, "Exact interpolation of band-limited functions," J. Appl. Phys., vol. 24, no. 12, pp. 1432-1436, Dec. 1953.

[7] R. J. Marks, Advanced Topics in Shannon Sampling and Interpolation Theory. New York: Springer-Verlag, 1992.

[8] A. J. Coulson, "A generalization of nonuniform bandpass sampling," IEEE Trans. Signal Processing, vol. 43, pp. 694-704, Mar. 1995.

[9] J. L. Brown, "Sampling rate reduction in multichannel processing of bandpass signals," J. Acoust. Soc. Amer., vol. 71, no. 2, pp. 378-383, 1982. 
[10] _ _ "On completeness and multichannel sampling," Signal Process., vol. 5, pp. 21-30, 1983.

[11] M. G. Beaty, "Multichannel sampling for multiband signals," Signal Process., vol. 36, pp. 133-138, 1994.

[12] A. Dabrowski, "Recovery of effective pseudopower in multirate signal processing," Ph.D. thesis, Poznan, 1988.

[13] _ "Signal reconstruction after heteromerous sampling, product modulation, and sampling rate alteration," in Proc. 18th Nat. Conf. Circuit Theory Electron. Syst., Zakopane, Poland, 1995.

[14] P. P. Vaidyanathan, Multirate Systems and Filter Banks. Englewood Cliffs, NJ: Prentice-Hall, 1993.

[15] P. P. Vaidyanathan and V. Liu, "Classical sampling theorems in the context of multirate and polyphase digital filter bank structures," IEEE Trans. Acoust., Speech, Signal Processing, vol. 36, pp. 1480-1495, Sept. 1988.

[16] _ "Efficient reconstruction of band-limited sequences from nonuniformly decimated versions by use of polyphase filter banks," IEEE Trans. Acoust., Speech, Signal Processing, vol. 38, pp. 1927-1938, Nov. 1990.

[17] Y. Lin and P. P. Vaidyanathan, "Periodically nonuniform sampling of a new class of bandpass signals," in Proc. 8th IEEE Digital Signal Processing Workshop, Leon, Norway, Sept. 1996, pp. 141-144.

[18] _ _ "Nonseparable sampling theorems for two-dimensional signals," in Proc. Int. Conf. Acoust., Speech, Signal Processing, Apr. 1996, vol. III, pp. 1969-1973.

[19] ㄴ. "Theory and design of two parallelogram filter banks," IEEE Trans. Signal Processing, vol. 44, pp. 1688-2706, Nov. 1996.

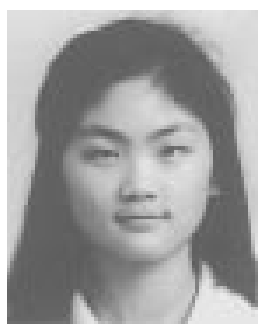

Yuan-Pei Lin (S'93-M'97) was born in Taipei, Taiwan, R.O.C., in 1970. She received the B.S. degree in control engineering from the National Chiao-Tung University, Hsinchu, Taiwan, in 1992 , and the M.S. and Ph.D. degrees, both in electrical engineering, from the California Institute of Technology, Pasadena, in 1993 and 1997, respectively.

She joined the Department of Control and Electrical Engineering of National Chiao-Tung University, in 1997. Her research interests include multirate filter banks, wavelets, and multidimensional signal processing.

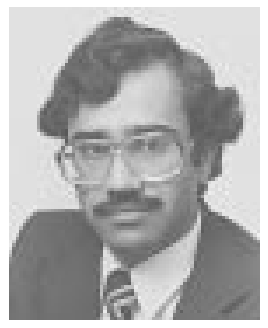

P. P. Vaidyanathan (S'80-M'83-SM'88-F'91) was born in Calcutta, India on Oct. 16, 1954. He received the B.Sc. (Hons.) degree in physics and the B.Tech. and M.Tech. degrees in radiophysics and electronics, all from the University of Calcutta, India, in 1974, 1977, and 1979, respectively, and the $\mathrm{Ph} . \mathrm{D}$. degree in electrical and computer engineering from the University of California at Santa Barbara (UCSB) in 1982.

He was a postdoctoral fellow at UCSB from September 1982 to March 1983. In March 1983 he joined the Electrical Engineering Department, California Institute of Technology, Pasadena, as an Assistant Professor, and since 1993 has been Professor of electrical engineering there. His main research interests are in digital signal processing, multirate systems, wavelet transforms, and adaptive filtering.

Dr. Vaidyanathan served as Vice-Chairman of the Technical Program committee for the 1983 IEEE International Symposium on Circuits and Systems, and as the Technical Program Chairman for the 1992 IEEE International Symposium on Circuits and Systems. He was an Associate Editor for the IEEE TRANSACTIONS ON CIRCUITS AND Systems from 1985 to 1987, and is currently an Associate Editor for the journal IEEE SIGNAL Processing LetTers, and a Consulting Editor for the journal Applied and Computational Harmonic Analysis.He has authored a number of papers in IEEE journals, and is the author of the book Multirate Systems and Filter Banks (Englewood Cliffs, NJ: Prentice-Hall, 1993). He has written several chapters for various signal processing handbooks. He was a recepient of the Award for excellence in teaching at the California Institute of Technology for the years 1983-1984, 1992-1993, and 1993-1994. He also received the NSF's Presidential Young Investigator award in 1986. In 1989 he received the IEEE ASSP Senior Award for his paper on multirate perfect-reconstruction filter banks. In 1990 he was recipient of the S. K. Mitra Memorial Award from the Institute of Electronics and Telecommunications Engineers, India, for his joint paper in the IETE Journal. He was also the coauthor of a paper on linear-phase perfect reconstruction filter banks in the IEEE TRANSACTIONS ON Signal Processing, for which the first author (T. Nguyen) received the Young Outstanding Author award in 1993. He was elected Fellow of the IEEE in 1991. He received the 1995 F. E. Terman Award of the American Society for Engineering Education, sponsored by Hewlett Packard Co., for his contributions to engineering education, especially the book Multirate Systems and Filter Banks. He was a Distinguished Lecturer for the IEEE Signal Processing Society for 1996-1997. 